\title{
Kaleidoscope laser
}

\author{
G. S. McDonald and G. P. Karman
}

Huygens Laboratory, Leiden University, Box 9504, 2300 RA Leiden, The Netherlands

\author{
G. H. C. New
}

Laser Optics and Spectroscopy Group, Department of Physics, Imperial College, London SW7 2BZ, UK

\author{
J. P. Woerdman
}

Huygens Laboratory, Leiden University, Box 9504, 2300 RA Leiden, The Netherlands

Received June 11, 1999

\begin{abstract}
We report the first calculations of mode patterns of unstable-cavity lasers with truly two-dimensional transverse geometries. A detailed account of numerical techniques, incorporating a nonorthogonal beampropagation method, and results for cavities with a range of transverse symmetries, such as regular polygonal and rhomboid, are presented. In view of the beautiful complexity of the eigenmodes predicted, a novel kaleidoscope laser is proposed. (C) 2000 Optical Society of America [S0740-3224(00)00803-1]

OCIS codes: $030.4070,050.1940,110.1220,120.2040,140.3410$.
\end{abstract}

\section{INTRODUCTION}

Hermite-Gaussian modes have formed the basis for the analysis and understanding of stable-cavity lasers for many decades. These modes tend to be highly paraxial and have low round-trip loss but often do not exploit the full volume of the gain medium. On the other hand, unstable-cavity lasers utilize diverging light wave fronts for high-gain extraction, leading to strong periodic aperturing effects and high-loss modes. ${ }^{1-3}$ The transverse structure of these modes is determined, to a large extent, by the detailed symmetry of the aperturing element, resulting in mode patterns that are radically different than those of conventional stable-cavity lasers.

To date, only unstable-cavity modes for onedimensional (1D) transverse geometries, and for rectangular and circular geometries in two dimensions, have been studied. ${ }^{3-12}$ This is because each of these configurations can be investigated by use of a $1 \mathrm{D}$ transverse calculation. We have undertaken, to our knowledge, the first investigation of the eigenmode patterns of unstablecavity lasers with truly two-dimensional (2D) aperture symmetries, concentrating specifically on resonators with rhombus and $N$-sided regular polygon aperture shapes. In this paper we present an account of the numerical techniques that we have developed for the calculation of these fully $2 \mathrm{D}$ mode profiles. Results for cavities with triangular, rhomboid, pentagonal, hexagonal, and octagonal aperture symmetries are reported. The profound, and indeed fractal, complexity of the mode patterns leads us to christen such devices "kaleidoscope lasers."

\section{THEORY}

The simple resonator configuration that we model is sketched in Fig. 1(a) and consists of a convex mirror (focal length $f$ ) and a concave mirror (focal length $f_{v}$ ) that are separated by a free space path of length $L$. For definiteness we model a confocal unstable resonator $\left(L \equiv L_{c}\right.$ $\left.=f_{v}-f\right)$ in which periodic aperturing of the circulating field occurs at the convex mirror. This aperturing may arise from the shape of the mirror itself or, more conveniently, be due to an aperture placed directly against this mirror. Figure 1(b) shows the aperture shapes that we consider, and it defines their relative sizes through the parameter $a$. The eigenmodes of the cavity are parametrized by only two variables, the round-trip magnification $M$ and the equivalent Fresnel number $N_{\text {eq }}$ given, for this particular configuration, by $M \equiv M_{c}=f_{v} / f$ and $N_{\text {eq }}$ $=(M-1) a^{2} / 2 \lambda L$. The same results can also be applied to different experimental configurations, albeit with different definitions of $M$ and $N_{\text {eq }}$. In general, the magnification is given $\mathrm{by}^{3} M=m+\left(m^{2}-1\right)^{1 / 2}$ (for a positive branch resonator), where ${ }^{12} m=\left(M_{c}+1 / M_{c}\right.$ - $\left.\delta^{2} / f f_{v}\right) / 2$ and a finite degree of nonconfocality has been introduced through $L=L_{c}+\delta$. The equivalent Fresnel number is given by ${ }^{3} N_{\mathrm{eq}}=a^{2}\left(M^{2}-1\right) / 2 \lambda B M$, where ${ }^{12}$ $B=L_{c}\left(1+M_{c}\right) / M_{c}+(2 f-\delta) \delta / f_{v}$ for aperturing at the convex mirror and $B=L_{c}\left(1+M_{c}\right)+\left(2 f_{v}+\delta\right) \delta / f$ when the aperture is at the other end of the resonator.

For rectangular and circular aperturing, semianalytical techniques are useful for determining an approximation to the mode profile. One of the more commonly used methods is the virtual source technique ${ }^{6}$ in which the cavity is unfolded into a sequence of effective apertures. The modal pattern is then constructed by taking a weighted sum of edge waves from each aperture and an undiffracted plane-wave component. At the heart of such methods is knowledge of the diffraction patterns that result from particular aperture shapes. However, it is quite surprising that there is almost no published work describing Fresnel diffraction at apertures with nonor- 


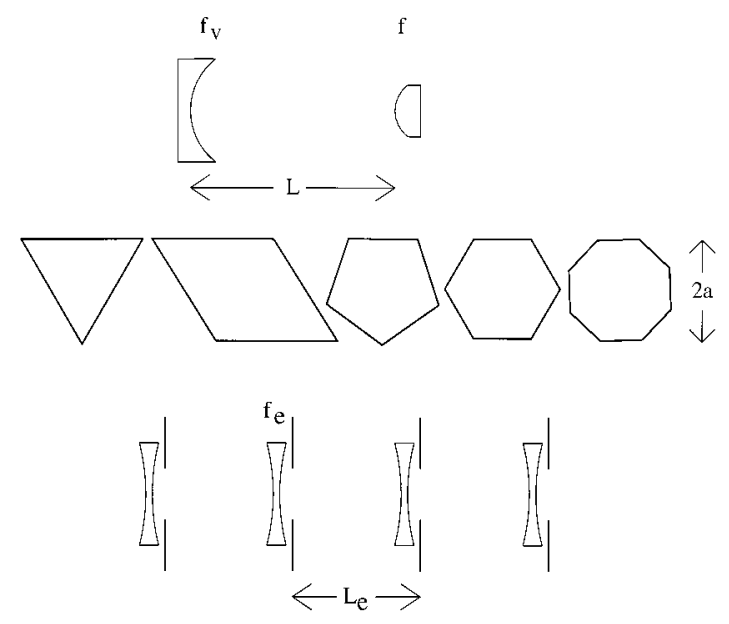

(a)

Fig. 1. Bare cavity configuration of the kaleidoscope laser. (a) Schematic picture of the unstable cavity, consisting of a convex mirror (focal length $f$ ) and a concave mirror (focal length $f_{v}$ ) separated by a distance $L$. The circulating field is apertured on each round trip by one of the mirrors or, equivalently, by an aperture placed directly against that mirror. (b) The transverse shapes of the apertures considered are plotted. (c) In the modeling we transform the above system to the equivalent lens guide sketched, which is a series of diverging lenses (focal length $f_{e}$ and a distance $L_{e}$ apart) and apertures adjacent to each of these lenses.

thogonal edges. A notable exception to this is Newton's two-knife experiment, ${ }^{13}$ which has only recently attracted some deserved attention. ${ }^{14}$ Newton reported that hyperbolic fringes appear in the diffraction patterns from thin wedge apertures as one moves toward the far-field domain. Nevertheless, the complexity of the aperture shapes considered here, and the absence of appropriate analytical work, render the generalization of existing semianalytical techniques quite involved; we therefore adopt a fully numerical approach.

In the numerical scheme used, the intracavity field is initialized with a plane wave and then evolved by circulation around the resonator by repeated application of a round-trip $A B C D$ matrix and aperturing. This mapping is iterated until the transverse profile converges, with sufficient accuracy, to the lowest-loss mode. Resolution of the aperture edges and the resulting diffraction patterns demands relatively dense discretization of the transverse plane. To minimize memory and run-time requirements of the simulations, we use a transverse basis that most closely matches the symmetry of the aperture shape. Figure 2 shows the geometry of the transverse computational axes in the most general case. For each transverse point, orthogonal and nonorthogonal coordinates are defined, $\left(x_{1}, y_{1}\right)$ and $\left(p_{1}, q_{1}\right)$, respectively. Denoting the angle between the $p-q$ axes by $\Phi$, we see that these sets of coordinates are related through $p_{1}$ $=x_{1}-y_{1} / \tan \Phi$ and $q_{1}=y_{1} / \sin \Phi$.

To evolve the field profile over each cavity round trip, we find it is advantageous to transform the actual confocal cavity to an equivalent lens guide, consisting of a sequence of concave lenses and apertures [Fig. 1(c)]. Requiring that this lens guide has the same value of $N_{\text {eq }}$ as the original cavity, we find the focal length of each lens to be $f_{e}=-L(M+1) /(M-1)^{2}$. The mode profile imme- diately before the concave lens in the equivalent guide $U_{e 1}(p, q)$ is related to the associated profile $U(p, q)$ approaching the convex mirror through

$$
\begin{aligned}
U_{e 1}(p, q)= & U(p, q) \\
& \times \exp \left[-i \pi \beta\left(p^{2}+q^{2}+2 p q \cos \Phi\right) / a^{2}\right],
\end{aligned}
$$

where $\beta=\left(a^{2} / \lambda L\right)(M-1) /(M+1)$. The defocusing and aperturing actions of the lens then transform the field to $U_{e 2}(p, q)$, where

$$
\begin{aligned}
U_{e 2}(p, q)= & U_{e 1}(p, q) \exp [-i \pi \beta(M-1) \\
& \left.\times\left(p^{2}+q^{2}+2 p q \cos \Phi\right) / a^{2}\right]
\end{aligned}
$$

inside the aperture and is zero otherwise. Free space propagation of $U_{e 2}(p, q)$ over a distance $L_{e}=L(1$ $+M) / M$ then yields $U_{e 3}(p, q)$ and completes the scheme for a single cavity transit.

The propagation step is implemented in the spatial frequency domain and thus involves a pair of fast Fourier transforms (FFT's). Although there exists a computationally faster scheme in which a cavity transit can be written in terms of only one $\mathrm{FFT}^{3}{ }^{3}$ we find that this latter scheme imposes an awkward constraint on the spatial grid. In 1D transverse calculations the effect of this constraint can be minimized by use of a very large number of transverse points, but in the $2 \mathrm{D}$ case this is not possible and the constraint ultimately limits the accuracy of the solution. We also find that the double FFT method permits a straightforward accommodation of a nonorthogonal transverse basis.

In terms of the Cartesian basis $x-y$ and the longitudinal coordinate $z$, propagation of the field involves the solution of

$$
\frac{\partial U(x, y)}{\partial z}=\frac{i}{2 k}\left(\frac{\partial^{2}}{\partial x^{2}}+\frac{\partial^{2}}{\partial y^{2}}\right) U(x, y)
$$

where $k$ is the free space propagation constant. In the nonorthogonal basis the field evolution is governed by

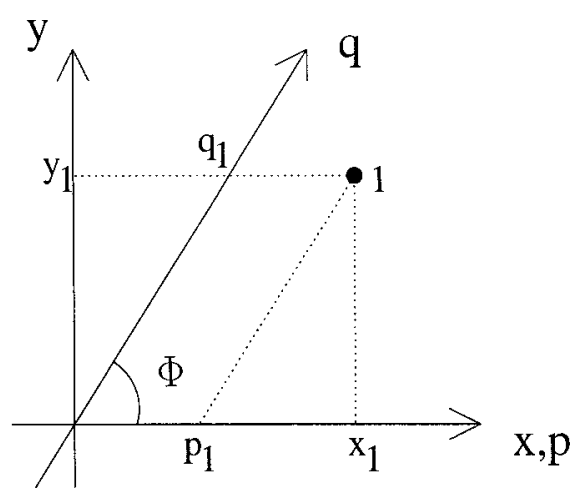

Fig. 2. Orthogonal $(x-y)$ and nonorthogonal $(p-q)$ axes in the transverse plane. An arbitrary point in this space, which is labeled 1 , has two pairs of coordinates, $\left(x_{1}, y_{1}\right)$ and $\left(p_{1}, q_{1}\right)$. 


$$
\begin{aligned}
\frac{\partial U(p, q)}{\partial z}= & \frac{i}{2 k \sin ^{2} \Phi} \\
& \times\left(\frac{\partial^{2}}{\partial p^{2}}+\frac{\partial^{2}}{\partial q^{2}}-2 \cos \Phi \frac{\partial}{\partial p} \frac{\partial}{\partial q}\right) U(p, q) .
\end{aligned}
$$

To employ FFT's for the solution of Eq. (4), we define a spectral function $\tilde{U}\left(K_{1}, K_{2}\right)$ such that

$$
\begin{aligned}
U(p, q)= & \int_{-\infty}^{\infty} \int_{-\infty}^{\infty} \tilde{U}\left(K_{1}, K_{2}\right) \\
& \times \exp \left[i\left(K_{1} p+K_{2} q\right)\right] \mathrm{d} K_{1} \mathrm{~d} K_{2},
\end{aligned}
$$

whereby free space propagation is effected by ${ }^{15}$

$$
\begin{aligned}
& \tilde{U}_{e 3}\left(K_{1}, K_{2}\right) \\
& =\tilde{U}_{e 2}\left(K_{1}, K_{2}\right) \\
& \quad \times \exp \left[-\frac{i L_{e}}{2 k \sin ^{2} \Phi}\left(K_{1}^{2}+K_{2}^{2}-2 \cos \Phi K_{1} K_{2}\right)\right] .
\end{aligned}
$$

Once a particular mode is established, the field profiles satisfy the self-consistency condition $U_{e 3}(p, q)$ $=\gamma U_{e 1}(p, q)$, where $\gamma$ is the (complex) mode eigenvalue. Equation (1) can then be inverted to determine the mode profile $U(p, q)$ in the actual cavity.

\section{RESULTS}

In recent experiments, ${ }^{9-11,16,17}$ unstable cavities with low $N_{\text {eq }}$ values and linear magnifications between 1 and 2 have been studied. We have concentrated our investigations of eigenmode patterns in this regime. To facilitate a comparison between transverse modes with different aperture shapes, we present results for a single value of magnification, ${ }^{17} M=1.3$.

First, we checked that the full $2 \mathrm{D}$ code gave results consistent with those of $1 \mathrm{D}$ codes for apertures with square and circular shapes. Second, since the transverse grid cannot sample individual edges of all polygon apertures in exactly the same fashion, checking the overall symmetry of each modal pattern proved to be a good test of whether we were using a sufficient number of transverse points. For $M=1.3$ we found that $256 \times 256$ transverse points were required for $N_{\text {eq }}$ up to 2.0 and that a grid density of $512 \times 512$ points was needed for higher values (up to $N_{\mathrm{eq}} \approx 4$ ). Higher-order corrections in the discretizations, which weight individual sampling points along the edges of each aperture, have also been employed, and the results obtained were checked against those presented here. Finally, the value of the Petermann $K$ factor (the so-called excess quantum-noise factor) for any particular eigenmode pattern depends very sensitively on the detail of its transverse structure, since the $K$ factor is the inverse of an almost vanishing modal overlap integral. $^{7}$ We used this quantity as a diagnostic to test the accuracy of $2 \mathrm{D}$ patterns using corresponding results from 1D calculations and to monitor the convergence of the field profiles. In common with studies of the $1 \mathrm{D}$ problem, ${ }^{12}$ we find that the number of cavity transits for convergence depends on the precise value of $N_{\text {eq }}$ considered and whether there is a nearby mode crossing (a point at which two eigenmodes of the cavity become degenerate).

Most graphical packages require data defined on a Cartesian basis, so it is convenient to transform the modal profiles $U(p, q)$ back to a rectilinear representation $U(x, y)$. To undertake this transformation using conventional interpolation techniques would be rather involved and may introduce inaccuracies. As an alternative, we use the Fourier transform shift property and perform the required transformation in the frequency domain. For each $q$, we define a function $\widetilde{U}(K)$ such that

$$
U(p)=\int_{-\infty}^{\infty} \tilde{U}(K) \exp (i K p) \mathrm{d} K,
$$

whereby $x=p+q \cos \Phi$ yields

$$
U(x)=\int_{-\infty}^{\infty} \tilde{U}(K) \exp (i K q \cos \Phi) \exp (i K p) \mathrm{d} K .
$$

Thus in practice, the reconstruction of $U(x, y)$ involves scanning through $q$ and, on each line of data, performing an FFT, applying a phase ramp given by $K q \cos \Phi$, and then using another FFT.

Column 1 of Fig. 3 shows results for triangular aperturing. The transverse intensity profiles of the modes have increasing complexity as $N_{\text {eq }}$ is increased from 0.5 to 4.0 (moving downward in the column). The values of $N_{\text {eq }}$ used were chosen to give an overview of the distinct patterns that arise as the aperture size is slowly increased. The choice of $N_{\text {eq }}$ values for other aperture shapes is made on the same basis. For $N_{\text {eq }}=0.5$, a single spot with overall triangular symmetry is evident. Closer inspection of this pattern reveals the presence of smooth curves connecting the bright regions that arise from the aperture corners. Since this modal pattern may be considered to be a superposition of aperture diffraction patterns that extend into the far field, we believe that these smooth contours are related to a generalization of Newton's result in which each aperture of the unfolded system gives rise to a combination of three wedge-type diffraction profiles.

The second profile in column 1 also displays a pattern that has bright spots from the aperture corners. This characteristic was found for all polygon mode profiles when $N_{\text {eq }} \approx 1$. As $N_{\text {eq }}$ is increased to 3.0 (the third triangular profile) a different type of eigenmode pattern is found that is composed of bright sides enclosing a nested structure. This profile is in fact similar to the Fresnel diffraction pattern of the closest aperture of the unfolded system. In the final plot of column 1 , where $N_{\text {eq }}=4.0$, the mode characteristics have changed yet again, and the pattern exhibits bright spots emanating from the aperture edges.

Our nonorthogonal computational technique is ideally suited to the modeling of configurations with rhomboid aperturing. In column 2 of Fig. 3 we show eigenmode profiles when the cavity aperture has the shape of a $60^{\circ}$ rhombus. For this configuration we show the cases $N_{\text {eq }}$ $=0.5,1.0,1.5$, and 3.0. Making a comparison with the modes for triangular aperturing, we clearly see that the 
same numerical value of $N_{\text {eq }}$ leads to much more developed modal structure. In fact, the parameter $a$ is defined such that, on the one hand, the rhombus aperture has twice the area of the corresponding triangular aperture but, on the other hand, has the same area as the corresponding square aperture. This highlights the somewhat arbitrariness in the definition of $N_{\mathrm{eq}}$. We define $a$ such that there is consistency with square and circular apertures, since in these cases $N_{\text {eq }}$ is formally defined. In doing so we regard a circle as a regular polygon with an infinite number of sides.

The equivalent Fresnel number actually has its origin in the relative phase of (magnifying and demagnifying) geometrical eigenmodes of the cavity and is distinct from the collimated Fresnel number $N_{c}=M a^{2} / B \lambda$. This latter parameter is more closely related to the number of diffraction ripples across the opening of the aperture ${ }^{3}$ and is generally larger than $N_{\text {eq }}$. This fact explains the relative complexity of modal patterns for such low values of $N_{\text {eq }}$. The rhomboid mode profiles for the lowest values of $N_{\text {eq }}$ considered confirm the generality of the brightcorners characteristic, and, with increasing $N_{\text {eq }}$, the mode can be seen to cycle through patterns with bright sides and nested structures. The final frame in column 2 displays a mode that is reminiscent of those found for square aperturing, ${ }^{11}$ even though the overall symmetry is rhomboid.

Eigenmode profiles for other types of polygonal aperturing are shown in columns $3-5$ of Fig. 3, where the transverse symmetry of the cavities are pentagonal, hexagonal, and octagonal, respectively. In each column a similar sequence of modal characteristics, as described earlier, is evident. For low $N_{\text {eq }}$ the modes become progressively more circular as the number of aperture sides is increased. For higher values of $N_{\text {eq }}$, it is interesting to note that the results are similar to snowflake patterns. This leads one to enquire whether the eigenmodes have an underlying fractal nature. Two of us have recently undertaken calculations of the fractal dimension of mode profiles of $1 \mathrm{D}$ and radially symmetric cavities. ${ }^{18}$ In these studies the dimension of the modes was found to lie between 1 and 2, confirming their fractal character. A simplified physical picture, involving the repeated magnification of the modal pattern on subsequent round trips, was also presented to support their findings. We note that this interpretation is essentially independent of the aper-
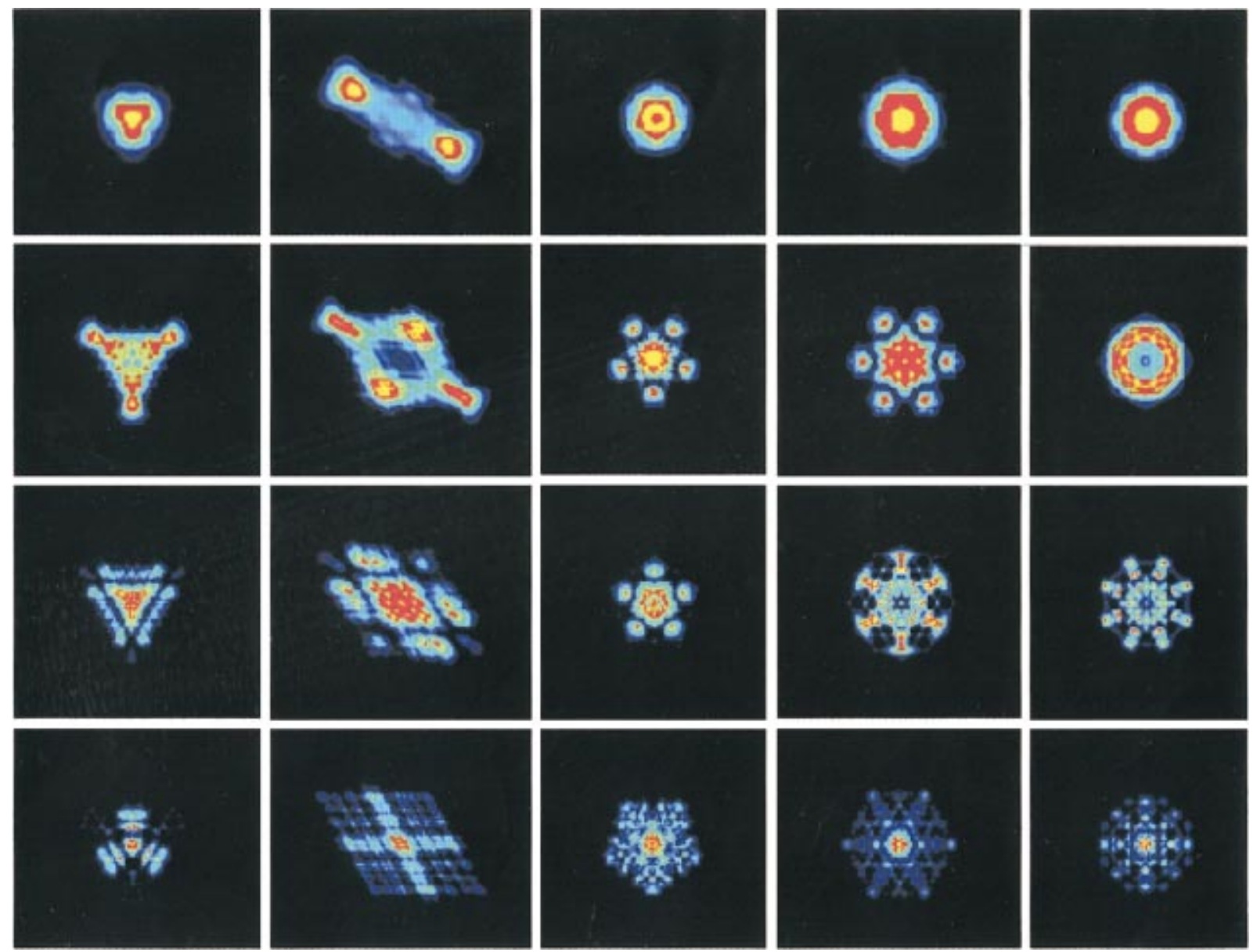

Fig. 3. Cross section of the transverse-mode profiles of the kaleidoscope laser. The defining aperture of the cavity has a different shape in each column of this figure. The profiles generally exhibit increasing complexity as the equivalent Fresnel number is increased (moving downward through each column). In column 1 the aperture has the shape of an equilateral triangle $\left(N_{\mathrm{eq}}=0.5,1.5,3.0\right.$, and 4.0$)$. Columns 2-5 are for rhomboid $\left(N_{\text {eq }}=0.5,1.0,1.5\right.$, and 3.0), pentagonal $\left(N_{\text {eq }}=0.5,1.5,2.0\right.$, and 4.0$)$, hexagonal $\left(N_{\text {eq }}=0.5,1.5,3.0\right.$, and $4.0)$, and octagonal aperturing $\left(N_{\text {eq }}=0.5,1.0,3.0\right.$, and 4.0), respectively. The colors yellow, red, green, blue, and black are used to represent different (decreasing) levels of light intensity. 
ture shape. On this basis the modes of cavities with polygonal and rhomboid symmetries are also expected to be fractal.

Since the fractal dimensions of $1 \mathrm{D}$ and square geometry modes are identical, we suspect that the modal dimension for each polygonal configuration also lies between 1 and 2 . It is therefore not surprising that any underlying fractal structure can be visualized in the transverse plane since this surface represents an embedding dimension of 2 (which is larger than the dimension of the pattern). To obtain accurate estimates of fractal dimension, one generally needs many thousands of distinct sampling points. Although this requirement can easily be met in $1 \mathrm{D}$ calculations, the $2 \mathrm{D}$ spectral method that we employ dictates that the modal structure occupies only a portion of the whole transverse grid, and this feature greatly reduces the number of distinct and useful data points that are available for quantitative dimension estimates.

\section{KALEIDOSCOPE LASERS}

Unstable-cavity lasers are usually associated with highpower applications. Here, we have proposed a novel kaleidoscope laser for which, particularly in the high $N_{\text {eq }}$ regime, dynamic tuning between modes and real-time adjustments of the shape and size of the aperture would result in stunning visual complexity. A prototype kaleidoscope laser ${ }^{17}$ has actually been constructed by ourselves using a miniature He-Xe laser. A simple technique, based on modifying commercial iris diaphragms, has been developed that yields the required (variable-size and variable-shape) apertures. Using the most sensitive diagnostic available (the Petermann $K$ factor), we have found excellent agreement between theoretical predictions and experimental results. ${ }^{17}$ Noting that the Fresnel number of a cavity depends on the aspect ratio (and not on the absolute size) of the system, the simplicity and the high-gain characteristics of the kaleidoscope laser geometry make it particularly suited to further miniaturization; relatively inexpensive and compact devices may be envisaged.

The original kaleidoscope device, invented by Sir David Brewster in 1816, essentially consists of just two plane mirrors that share a common edge. ${ }^{19}$ The angle $\theta$ between these mirrors defines the $N$-fold polygonal symmetry of the patterns produced through the relation $N$ $=2 \pi / \theta$. A formal connection with the kaleidoscope laser can be established by employing Babinet's principle and decomposing an $N$-sided polygon aperture of the unstable cavity into $N$ congruent isosceles triangles. The internal angle of each of those component triangles, $2 \pi / N$, then corresponds to the angle between the mirrors of Brewster's kaleidoscope.

This suggests that kaleidoscope mirrors themselves could be employed as an optical element and could give rise to novel laser designs. They could impose polygonal transverse boundary conditions and thus replace more complex aperture assemblies. The result would be a laser whose modes could be easily scanned through an infinite number of possible polygonal symmetries (by vari- ance of the angle $\theta$ ). Alternatively, other fixed-symmetry systems could be built; gain rods whose longitudinal surfaces were polished or more exotic lasers that exploit a three-mirror kaleidoscope tube are possible.

\section{SUMMARY}

We have reported the first calculations, to our knowledge, of unstable-cavity eigenmodes for configurations with truly 2D transverse geometries. Modal patterns for aperturing with triangular, rhomboid, pentagonal, hexagonal, and octagonal symmetries have been mapped out as a function of the equivalent Fresnel number of the cavity. In contrast to the lowest-loss modes of stable-cavity lasers, a rich and beautiful complexity is revealed that suggests such systems be termed kaleidoscope lasers.

The use of a nonorthogonal computational grid made an important contribution to the analysis. Although this technique ${ }^{15}$ has received little attention since it was first proposed in 1993, it is clearly a very powerful and flexible method with a wide range of potential applications. These include the modeling of tapered optical structures and, more generally, two-and three-dimensional propagation problems that involve nonorthogonal features and where efficient discretization is a necessity. Moreover, there is almost no computational overhead arising from undertaking propagation calculations in a nonorthogonal frame.

We plan further theoretical and experimental investigations of kaleidoscope lasers, including alternative cavity designs, the study of more general aperture shapes, and considerations of higher-order modes. ${ }^{12}$ This will lend more insight into general Fresnel diffraction problems and the character of excess quantum noise, as well as permit a fuller exploration of the diversity of modal patterns that are possible.

\section{ACKNOWLEDGMENTS}

The authors acknowledge numerous invaluable discussions with M. van Eijkelenborg and T.I.J. Albaho, as well as interaction over many years with A. E. Siegman. The project was supported by the Foundation for Fundamental Research of Matter of the Netherlands, ESPRIT contract 20029 (ACQUIRE), TMR contract ERB4061PL951021 (Micro-Lasers and Cavity QED) and the UK Engineering and Physical Sciences Research Council under grant GR/L90583.

Please send correspondence to G. H. C. New, Laser Optics and Spectroscopy Group, Department of Physics, Imperial College, London SW7 2BZ, UK. Telephone: +44 207594 7791. Fax: +44 207823 8376. E-mail: g.new@ic.ac.uk.

\section{REFERENCES}

1. A. E. Siegman, "Unstable optical resonators," Appl. Opt. 13, 353-367 (1974).

2. J. Arnaud, Beam and Fiber Optics (Academic, New York, 1976). 
3. A. E. Siegman, Lasers (Oxford University, London, 1986).

4. P. Horwitz, "Asymptotic theory of unstable resonator modes," J. Opt. Soc. Am. 63, 1528-1543 (1973).

5. M. A. Lauder and G. H. C. New, "Biorthogonality properties and excess noise factors of unstable optical resonators," Opt. Commun. 31, 343-348 (1979).

6. W. H. Southwell, "Unstable-resonator-mode derivation using virtual-source theory,” J. Opt. Soc. Am. A 3, 1885-1891 (1986).

7. G. H. C. New, "The origin of excess noise," J. Mod. Opt. 42 , 799-810 (1995).

8. M. A. Rippin and G. H. C. New, "Excess noise factors in circular unstable resonators," J. Mod. Opt. 43, 993-1008 (1996).

9. M. A. van Eijkelenborg, A. M. Lindberg, M. S. Thijssen, and J. P. Woerdman, "Resonance of quantum noise in an unstable cavity laser," Phys. Rev. Lett. 77, 4314-4317 (1996).

10. M. A. van Eijkelenborg, A. M. Lindberg, M. S. Thijssen, and J. P. Woerdman, "Unstable-resonator diffraction losses and the excess-noise factor," Phys. Rev. A 55, 4556-4562 (1997).

11. M. A. van Eijkelenborg, A. M. Lindberg, M. S. Thijssen, and J. P. Woerdman, "Higher-order transverse modes of an unstable-cavity laser," IEEE J. Quantum Electron. 34, 955-965 (1998).
12. G. S. McDonald, J. P. Woerdman, and G. H. C. New, "Excess noise in low Fresnel number unstable resonators," Opt. Commun. 164, 285-295 (1999).

13. Sir Isaac Newton, Opticks, 4th ed. (London, 1730, reprinted by Dover, New York, 1952).

14. M. P. Silverman and W. Strange, "The Newton two-knife experiment: intricacies of wedge diffraction," Am. J. Phys. 64, 773-787 (1996).

15. G. S. McDonald and W. J. Firth, "Spatial grid symmetries and reduced models in the simulation of beam counterpropagation in a nonlinear medium," J. Mod. Opt. 40, 23-32 (1993).

16. Y. J. Cheng, C. G. Fanning, and A. E. Siegman, "Experimental observation of a large excess quantum noise factor in the linewidth of a laser oscillator having nonorthogonal modes," Phys. Rev. Lett. 77, 627-630 (1996).

17. G. P. Karman, G. S. McDonald, J. P. Woerdman, and G. H. C. New, "Excess-noise dependence on intra-cavity aperture shape," Appl. Opt. 38, 6874-6878 (1999).

18. G. P. Karman and J. P. Woerdman, "Fractal structure of eigenmodes of unstable-cavity lasers," Opt. Lett. 23, 19091911 (1998).

19. J. Walker, "The amateur scientist," Sci. Am. 253, 124-130 (1985) 